\title{
La Forêt romantique, études réunies par Vigor caillet
}

\author{
Michel Arrous
}

\section{(2) OpenEdition}

\section{Journals}

\section{Édition électronique}

URL : http://journals.openedition.org/studifrancesi/2178

DOI : 10.4000/studifrancesi.2178

ISSN : 2427-5856

\section{Éditeur}

Rosenberg \& Sellier

\section{Édition imprimée}

Date de publication : 1 avril 2014

Pagination : 153-154

ISSN : 0039-2944

\section{Référence électronique}

Michel Arrous, «La Forêt romantique, études réunies par Vigor caillet », Studi Francesi [En ligne], 172

(LVIII | I) | 2014, mis en ligne le 01 avril 2014, consulté le 18 septembre 2020. URL : http://

journals.openedition.org/studifrancesi/2178; DOI : https://doi.org/10.4000/studifrancesi.2178

Ce document a été généré automatiquement le 18 septembre 2020.

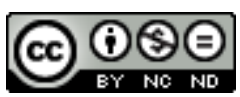

Studi Francesi è distribuita con Licenza Creative Commons Attribuzione - Non commerciale - Non opere derivate 4.0 Internazionale. 


\title{
La Forêt romantique, études réunies par Vigor caillet
}

\author{
Michel Arrous
}

\section{RÉFÉRENCE}

AA. VV., La Forêt romantique, études réunies par Vigor CAILLET, Bordeaux, Presses

Universitaires de Bordeaux, 2012, «Eidôlon», n. 103, pp. 275.

2012

1 Espace sauvage évolutif et soumis aux lois de l'économie, la forêt a sa part de barbarie ou de bestialité, de mystère aussi, dont le romantisme s'est nourri dans un attachement où se mêlent sacralisation, nostalgie et sensibilité écologique. L'univers sylvestre avec ses enchantements et ses paradoxes, déjà présents dans l'épisode bellifontain (Musset, Sand, Flaubert, Maupassant) perdure jusque dans l'imaginaire «fin-de-siècle». Dans son avant-propos (pp. 8-18), Vigor CAILLET dit justement que la forêt «a constitué un espace d'élection pour le $\mathrm{XIX}^{\mathrm{e}}$ siècle».

2 La forêt n'a bien sûr pas attendu Chateaubriand pour être romantique; néanmoins la Muse des bois lui a inspiré une nouvelle esthétique descriptive où se rejoignent les motifs traditionnels de la forêt antique, pagano-celtique et biblique. Sébastien BAUDOIN retrace ce polymorphisme de l'expérience sylvestre (Des bois de Combourg aux forêts américaines, transfigurations du modèle sylvestre dans l'œuvre de Chateaubriand, pp. 19-28). À cet enchantement de la forêt on peut opposer l'univers horizontal d'un peintreromancier: Jérôme LAURENT («L'ombre bleue des grands arbres»: les arbres et la forêt dans "Dominique” d'Eugène Fromentin, pp. 29-36) rappelle, après Thibaudet, que dans ce roman les forêts ont laissé place aux bois taillés et aux arbres plantés qui font écran entre la nature sauvage et l'intimité domestique. Un autre aspect de la thématique de la forêt, monde neuf, exotique et ensorcelant, investi de la sensibilité des personnages, est abordé par Fabienne MARIÉ LIGER (La représentation de la forêt comme confrontation de l'individu avec lui-même dans plusieurs récits russes du XIX siècle, pp. 37-49). Chez 
Pouchkine (Eugène Onéguine), Tolstoï (Les Cosaques), Gontcharov (La Falaise), l'exploration de la nature et de la forêt suscite l'exploration d'une personnalité et l'interrogation sur la société qui l'entoure. Ce pouvoir de la forêt, Christian CHELEBOURG le cerne par une approche phénoménologique du surnaturalisme romantique (Poétique romantique de la forêt magique, pp. 52-63), dense investigation qui explique, à partir de Sand, Dumas, Hugo et Nodier, comment les romantiques ont fait émerger une nouvelle représentation de la forêt en en faisant «un véritable opérateur de fantastique». Le romantisme «surnaturalise» la forêt qui est «dynamisée par l'expression de la passion et du désir physique». Barbara CISE, dans un aperçu de sa thèse en cours sur l'imaginaire surnaturaliste de Balzac, montre que chez le romancier ce lieu naturel qu'est la forêt, temple sacré, permet de sentir le surnaturel («Les merveilles de la forêt» balzacienne, pp. 65-76). On retrouve des liens de communication aussi forts, réels ou imaginaires: Martine REID (Les forêts de George Sand, pp. 77-88) étudie les occurrences sandiennes de la forêt à partir d'un échantillonnage thématique prélevé aussi bien dans les textes autobiographiques que dans les romans, où on lit à la fois l'histoire de la nature et son appropriation par l'homme, mais aussi l'apport des récits légendaires. Au sein de ce paysage de la «littérature forestière», Daudet occupe une place bien à lui, avec ses forêts à la fois sauvages et humanisées. Sa pratique pointilliste ou impressionniste se distingue de celle des Goncourt ou de Flaubert. De plus, et Pierre-Jean DUFIEF le met en évidence (Les forêts d'Alphonse Daudet, pp. 89-98), il y a chez lui une vision binaire: la forêt peut être le théâtre de la plus extrême cruauté et un lieu poétisé. La forêt peut être aussi un lieu hostile à la civilisation. Andrée MANSAU le rappelle (Angoisse et peur de la mort dans la forêt de Shakespeare / Walter Scott à George Sand, pp. 99-108): on retrouve, dans la Ballade de la reine Mab (1842), ces êtres maléfiques tout droit venus de Macbeth, de La Fiancée de Lamermoor ou de Roméo et Juliette de Berlioz. De même pour La Petite Fadette ou Le Marquis de Villemer, œuvres qui ne sont peut-être pas aussi assagies qu'on le dit, où la forêt que traversent Fadette et Caroline est le lieu d'une épreuve angoissante. S'il y a un endroit fort pauvre en arbres, c'est bien la demeure familiale de Vigny où s'est déroulé en juin 2010 le colloque «La forêt romantique», lieu que Laurent JOCELYN-LAFFONT a associé aux adaptations théâtrales de La Petite Fadette (en 1850 et 1860) (La forêt romantique au Manoir du Maine Giraud. "Chants dans la forêt», pp. 109-117), brève digression montrant comment l'opéra édulcore aussi bien la campagne berrichonne qu'une légende bretonne orchestrée par Meyerbeer, à partir de Souvestre et de La Villemarqué.

3 Les romantiques se sont emparés de la forêt de Fontainebleau qui finit par devenir un poncif. Les clichés romantiques ne manquent pas dans les quatre versions de l'épisode fameux du séjour de Sand et Musset au cœur du massif en 1833. À partir des récits de Musset, Sand, Paul de Musset et Louise Collet, Sébastien MADRIASSE relève les motifs, traits et clichés romantiques de ces évocations ambiguës qui ont fait des gorges de Franchard «un lieu mythique, symbole de la passion amoureuse mais aussi du Romantisme lui-même» (Alfred de Musset et l'épisode de Fontainebleau: une poétique de la forêt romantique entre merveilleux, sacralisation et ironie, pp. 119-132). Quant au séjour de Frédéric et Rosanette, lequel a pour intertexte l'épisode Sand-Musset, si l'idylle et ses attendrissements propres à la tradition romantique font oublier le désarroi provoqué par l'Histoire, Patrick FEYLER montre avec précision comment il échappe au romantisme par l'ironie (Nostalgie, dérision et dépassement du Romantisme: la forêt de Fontainebleau dans "L'Éducation sentimentale" de Flaubert, pp. 133-146). Le poncif bellifontain n'a pas rebuté 
Maupassant qui a choisi pour décor de son dernier roman ce motif topographique. Catherine BOTTEREL-MICHEL s'interroge sur le traitement de l'illusion référentielle (L'épisode bellifontain dans "Notre Cour" de Maupassant: réécriture et originalité d'un topos, pp. 147-157). On assiste à une «déflaubertisation» de Maupassant qui veille à s'écarter du maître en préférant dans ses emprunts la signification symbolique de la forêt à sa représentation réaliste.

4 Depuis le Moyen Âge, la forêt est un élément de la poétique de la rêverie à valeur d'épreuve ou de refuge, lieu de relégation sociale et de rédemption spirituelle qui caractérise aussi la forêt romantique. Dans ce paysage originaire on fait l'expérience fondamentale du sublime et du ressourcement, chez Gautier, chez Stendhal (voir la forêt de la Faggiola dans L'Abbesse de Castro dont le pouvoir énergétique aurait mérité une analyse), et chez Tocqueville (Voyage en Amérique) qui perçoit dans la forêt une manifestation du vouloir-vivre universel. C'est ce chaos d'une inquiétante étrangeté qu'appréhende Yvon LE SCANFF (Forêt verte et forêt noire: une polarité romantique, pp. 159-171).

Les ambiguïtés féminines de l'espace sylvestre sont évidemment présentes dans la littérature décadente. Morgane LERAY (Perdons-nous dans les bois, voir si la louve n'y est pas: féminité et érotisme dans la forêt fin-de-siècle, pp. 173-181) et Vigor CAILLET ("C'est le truc par où on monte les arbres!» Les dessous de la forêt dans "Les Hors Nature" de Rachilde, pp. 183-199), ont choisi un lieu commun du décadentisme. On retrouve dans Méphistophéla de Catulle Mendès (1890) la tradition fantasmatique de la forêt comme épiphanie de la féminité, une forêt qui est le lieu de l'initiation sensuelle en servant d'écrin aux amours saphiques; de même chez Rachilde, non sans surenchère, avec l'embrasement artificiel et l'épuisement des sens face à la persistance de l'univers naturel.

Le fait que la forêt si présente dans le roman gothique n'ait été mentionnée que comme source d'inspiration pour les romantiques rehausse l'intérêt de la communication illustrée et indispensable de Lauric GUILLAU qui invite à redécouvrir un artiste oublié par la critique française (La forêt gothique chez Gustave Doré, pp. 201-215). Dans l'œuvre de Doré (1832-1883), la forêt et sa vision cosmique du paysage (inspirée de John Martin et de Turner) constituent un motif récurrent au centre de son romantisme noir, quelque peu décalé dans une époque vouée au réalisme. La selva oscura primitive qui caractérise le paysage doréen a influencé les illustrateurs de Jules Verne, les peintres angloaméricains du sublime et le cinéma. Les peintres et les primitifs de la photographie font l'objet d'une étude historique et esthétique par Hélène SORBE (Peinture d'arbres autour de Barbizon, panthéisme romantique et photogénie de la forêt, pp. 217-235). La variété de paysages qu'offre la forêt de Fontainebleau va attirer les peintres; en même temps, la conception de l'arbre évolue, de la pratique éclectique classique qui le reléguait aux marges du tableau à sa représentation théâtralisée qui privilégie la nature animée et fait de lui le sujet du tableau. Peintres et photographes œuvrant d'ailleurs pour la conservation de la forêt.

7 La dimension iconographique n'a pas été oubliée puisque ce volume consacré aux realia et à l'imaginaire de la forêt se clôt par un portfolio militant composé des clichés du photographe et planteur d'arbres Alain GuILLou (pp. 237-270). À propos de l'imaginaire de la forêt, un petit regret néanmoins: auraient pu être évoqués Le Roman de la forêt d'Ann Radcliffe (1791) et, à titre anecdotique, Victor ou l'Enfant de la forêt de F. G. DucrayDuminil (1797). 